\title{
Whole pelvic helical tomotherapy for locally advanced cervical cancer: technical implementation of IMRT with helical tomothearapy
}

\author{
Chen-Hsi Hsieh ${ }^{1,3}$, Ming-Chow Wei², Hsing-Yi Lee ${ }^{1}$, Sheng-Mou Hsiao ${ }^{2}$ \\ Chien-An Chen ${ }^{1}$, Li-Ying Wang7, Yen-Ping Hsieh ${ }^{8}$, Tung-Hu Tsai, ${ }^{3,9}$, \\ Yu-Jen Chen*3,4,5,6 and Pei-Wei Shueng*1,10,11
}

Address: ${ }^{1}$ Department of Radiation Oncology, Far Eastern Memorial Hospital, Taipei, Taiwan, ${ }^{2}$ Departments of Obstetrics and Gynecology, Far Eastern Memorial Hospital, Taipei, Taiwan, ${ }^{3}$ Institute of Traditional Medicine, School of Medicine, National Yang-Ming University, Taipei, Taiwan, ${ }^{4}$ Department of Radiation Oncology, Mackay Memorial Hospital, Taipei, Taiwan, ${ }^{5}$ Department of Medical Research, Mackay Memorial Hospital, Taipei, Taiwan, ${ }^{6}$ Graduate Institute of Sport Coaching Science, Chinese Culture University, Taipei, Taiwan, ${ }^{7}$ School and Graduate Institute of Physical Therapy, College of Medicine, National Taiwan University, Taipei, Taiwan, ${ }^{8}$ Department of Healthcare Administration, Asia University, Taichung, Taiwan, ${ }^{9}$ Department of Education and Research, Taipei City Hospital, Taipei, Taiwan, ${ }^{10}$ Department of Radiation Oncology, National Defense Medical Center, Taipei, Taiwan and ${ }^{11}$ General Education Center, Oriental Technology Institute, Taipei, Taiwan

Email: Chen-Hsi Hsieh - chenci28@ms49.hinet.net; Ming-Chow Wei - wei@mail.femh.org.tw; Hsing-Yi Lee - nefertari1204@yahoo.com.tw; Sheng-Mou Hsiao - smhsiao2@gmail.com; Chien-An Chen - kenk102000@yahoo.com.tw; Li-Ying Wang - liying@ntu.edu.tw; YenPing Hsieh - fannyhsieh@hotmail.com; Tung-Hu Tsai - thtsai@ym.edu.tw; Yu-Jen Chen* - chenmdphd@yahoo.com; PeiWei Shueng* - shueng@hotmail.com

* Corresponding authors

Published: 10 December 2009

Radiation Oncology 2009, 4:62 doi:10.1186/1748-717X-4-62
Received: 22 September 2009

Accepted: 10 December 2009

This article is available from: http://www.ro-journal.com/content/4/I/62

(C) 2009 Hsieh et al; licensee BioMed Central Ltd.

This is an Open Access article distributed under the terms of the Creative Commons Attribution License (http://creativecommons.org/licenses/by/2.0), which permits unrestricted use, distribution, and reproduction in any medium, provided the original work is properly cited.

\footnotetext{
Abstract

Background: To review the experience and to evaluate the treatment plan of using helical tomotherapy $(\mathrm{HT})$ for the treatment of cervical cancer.

Methods: Between November Ist, 2006 and May 3I, 2009, 10 cervical cancer patients histologically confirmed were enrolled. All of the patients received definitive concurrent chemoradiation (CCRT) with whole pelvic HT (WPHT) followed by brachytherapy. During WPHT, all patients were treated with cisplatin, $40 \mathrm{mg} / \mathrm{m}^{2}$ intravenously weekly. Toxicity of treatment was scored according to the Common Terminology Criteria for Adverse Events v3.0 (CTCAE v3.0).

Results: The mean survival was 25 months (range, 3 to 27 months). The actuarial overall survival, diseasefree survival, locoregional control and distant metastasis-free rates at 2 years were $67 \%, 77 \%, 90 \%$ and $88 \%$, respectively. The average of uniformity index and conformal index was 1.06 and I.19, respectively. One grade 3 of acute toxicity for diarrhea, thrombocytopenia and three grade 3 leucopenia were noted during CCRT. Only one grade 3 of subacute toxicity for thrombocytopenia was noted. There were no grade 3 or 4 subacute toxicities of anemia, leucopenia, genitourinary or gastrointestinal effects. Compared with conventional whole pelvic radiation therapy (WPRT), WPHT decreases the mean dose to rectum, bladder and intestines successfully.

Conclusion: HT provides feasible clinical outcomes in locally advanced cervical cancer patients. Longterm follow-up and enroll more locally advanced cervical carcinoma patients by limiting bone marrow radiation dose with WPHT technique is warranted.
} 


\section{Background}

Cervical cancer is the second most frequent cancer among women worldwide [1]. It has demonstrated the superiority of combined chemotherapy with radiotherapy (RT) in the treatment of advanced cervix cancer [2,3]. The radiation therapy consists of external beam irradiation to the primary tumor and corresponding region of lymphatic drainage, followed by brachytherapy to boost the gross tumor in the cervix. A significant benefit of chemoradiation on both overall survival and progress-free survival rate was mentioned [4]. However, grade 3 or 4 haematological (white cell count, 16\% vs. $8 \%$; platelets, $1 \cdot 5 \%$ vs. $0 \cdot 2 \%$; haematological not otherwise specified, $29 \% v$ s. $1 \%$ ) and gastrointestinal toxicities ( $9 \%$ vs. 4\%) significantly greater in the concomitant chemoradiation group than the RT alone group should also be mentioned. Tan et al. [5] also proposed a late toxicity observation for concomitant chemoradiation of locally advanced cervical cancer. There were $14.5 \%, 9.4 \%$ and $11.4 \%$ for grade 3 or 4 urinary, bowel and affecting other organs complications, respectively.

With the advances in radiotherapy modalities, whole pelvic intensity-modulated radiotherapy (WP-IMRT) applied to gynecologic malignancies with excellent planning target volume (PTV) coverage and is associated with less acute gastrointestinal sequelae than conventional whole pelvic radiotherapy (WPRT) as reported by Mundt et al. [6]. Under similar target coverage, IMRT is superior to conventional techniques in normal tissue sparing for the treatment of cervical cancer and a number of groups have explored IMRT in the gynecologic setting as a method to minimize the gastrointestinal, genitourinary, and bone marrow toxicity that occurs in conventional RT [7-11].

Helical tomotherapy (HT) is a new CT-based rotational intensity modulated radiotherapy and provides an impressive ability for highly conformal dose distributions and simultaneous critical organ sparing ability $[12,13]$. HT is being tested to apply for gynecologic malignancies recently and provides encouraging results about excellent setup accuracy and reducing margins for the external beam treatment of gynecologic malignancies [14]. However, this report did not provide the clinical results about the gynecologic malignancies treated by HT.

In our institute, a Tomotherapy Hi-Art system (Tomotherapy, Inc., Madison, Wisconsin, USA) was installed and used for treatment from November 2006. We report here our initial clinical 2 years experience for patients with locally advanced cervical cancer with HT, focusing on the correlation between dosimetry, clinical outcome and early toxicities.

\section{Methods \\ Patient's characteristics}

Between November 1st, 2006 to May 31, 2009, 10 patients undergoing whole pelvic HT (WPHT) for locally advanced cervical cancer without pelvic or paraarotic lymphadenopathy at Far Eastern Memorial Hospital (FEMH) were retrospectively enrolled. Staging investigations included complete history and physical examination, fiberoptic endoscopic evaluation, complete blood counts, liver and renal function tests, chest X-ray, magnetic resonance imaging (MRI) scans or computed tomography (CT) scans of the pelvic region. The disease was staged according to the International Federation of Gynecology and Obstetrics (FIGO) criteria [15].

\section{Radiotherapy}

Radiotherapy was administered to the whole pelvic region in 28 fractions totaling 50.4 Gy followed by intracavitary brachytherapy. The total dose of brachytherapy delivered was $30 \mathrm{~Gy} / 6$ fractions in patients. The total dose delivered to point A (a reference location $2 \mathrm{~cm}$ lateral and $2 \mathrm{~cm}$ superior to the cervical os) was $80.4 \mathrm{~Gy}$ in patients; the total dose delivered to point $\mathrm{p}$ (the pelvic wall) was 55.0 Gy in patients. Cisplatin (CDDP) was administered during external radiation, beginning on the first day of radiation for 5 weeks concurrent with WPHT. A dose of $40 \mathrm{mg} /$ $\mathrm{m}^{2}$ CDDP (maximum dose, $70 \mathrm{mg}$ ) was used and administered via a peripheral vein to patients.

\section{Immobilization}

A BlueBAG ${ }^{\text {тм }}$ immobilization system (Medical Intelligence, Schwabmünchen, Germany) was used for each of these patients to fix pelvic and extremities. Positioning was supine with arms up, and feet placed in an ankle holder. All patients underwent a CT planning scan with our departmental scanner (Siemens Somatom Plus 4 CT scanner) from the diaphragm to $5 \mathrm{~cm}$ below the ischial tuberosities. Localization marks were placed on anterior and lateral sides of the patients at the mid-plane and midline at the level of L4-L5 vertebral body interspace. CT with 5-mm slice thickness was taken for treatment planning. Target objects and normal structures were contoured on a Pinnacle3 treatment planning system (Philips Healthcare, Madison, Wisconsin, USA). The MRI or CT images were retrieved on a Pinnacle workstation and fused with the CT images for contouring of the tumor volume.

\section{Delineation of target volumes}

Delineation and constraints was according to Radiation Therapy Oncology Group (RTOG) 0418 protocol and the International Commission on Radiation Units and Measurements reports 50 [16] and 62 [17] recommendations. The Gross Tumor Volume (GTV) was defined as all known 
gross disease determined from CT, clinical information, and MRI. The Clinical Target Volume (CTV) was defined as areas considered containing potential microscopic disease. Internal Target Volume (ITV) was defined as the volume of the vagina and paravaginal soft tissues that is in both the empty and full bladder CT scans that were done at the time of simulation and fused together. The Planning Target Volume (PTV) would provide a $7 \mathrm{~mm}$ margin (anteriorly, posteriorly, laterally, as well as in the superior and inferior directions) around the nodal CTV and ITV. The treatment plan would be done on the full bladder scan. The treatment plan used for each patient would be based on an analysis of the volumetric dose, including dose volume histogram (DVH) analyses of the PTV and critical normal structures. The GTV plus a 7-mm expansion was defined as the primary tumor CTV to account for microscopic spread, excluding the bowel, bladder, and rectum if they were not clinically involved); The nodal CTV should include the internal (hypogastric and obturator), external, common iliac lymph nodes perinodal tissue, pertinent clips and down to the level of S3. Identification of the CTV usually began with the identification of the iliac vessels. The average margin would be 7 $\mathrm{mm}$. Bone and intraperitoneal small bowel should be excluded from the CTV; also, iliopsoas muscle that lies adjacent to clinically negative lymph nodes should also be excluded from the CTV. Approximately $1.5 \mathrm{~cm}$ of tissue anterior to the S1, S2 and S3 sacral segments was usually added to the CTV in order to include the presacral lymph nodes and uterosacral ligaments. The most antero-lateral external iliac lymph nodes that lied just proximal to the inguinal canal should be excluded from the CTV. The CTV of the nodes should end $7 \mathrm{~mm}$ from L4/L5 interspace to account for the PTV. The PTV for nodes stopped at L4/L5 interspace. The vaginal and parametrial CTV should actually be an ITV, which will account for internal organ motion. The inferior limit was usually around the level of the upper third of the symphysis pubis but could be individualized based on inferior spread of the patient's tumor. The lateral margin of the vaginal PTV should be to the obturator muscle. However, at least $3 \mathrm{~cm}$ of the vagina needed to be treated or at least $1 \mathrm{~cm}$ below the obturator foramen. The $90 \%$ isodose surface covered between $95 \%$ and $98 \%$ of the PTV 50.4, or volumes of overdose exceed $115 \%<5 \%$ of the PTV 50.4 volume could be considered acceptable. The field width, pitch, and modulation factor (MF) usually used for the WPHT treatment planning optimization were $2.5 \mathrm{~cm}, 0.32$ and 3.0 , respectively. All patients received daily megavoltage computed tomography (MVCT) acquisitions for setup verification [18].

Normal structures will be contoured using the full-bladder CT scan. The OARs (i.e., bladder, rectum, sigmoid, small bowel, and femoral heads) were contoured as solid organs. Dose-volume constraints for normal tissues were as follows: small bowel $(2 \mathrm{~cm}$ above the most superior vessel contour) $<30 \%$ to receive $\geq 40 \mathrm{~Gy}$, minor deviation $30 \%$ to $40 \mathrm{~Gy}$; Rectum $<60 \%$ to receive $\geq 30 \mathrm{~Gy}$, minor deviation $35 \%$ to $50 \mathrm{~Gy}$; Bladder $<35 \%$ to receive $\geq 45$ Gy, minor deviation $35 \%$ to $50 \mathrm{~Gy}$; Femoral head $\leq 15 \%$ to receive $\geq 30 \mathrm{~Gy}$, minor deviation $20 \%$ to $30 \mathrm{~Gy}$.

\section{Intracavitary brachytherapy}

An iridium-192 (high-dose-rate) source was used with standard Fletcher-Suit-Delclos intracavitary applicators. Patients were treated twice a week after WPHT completed for 3 weeks, with a prescribed dose of $500 \mathrm{cGy}$ per fraction to Point A. The high-dose rate (HDR) source dwell times were manually calculated based on our institutional system of empiric intracavitary irradiation rules. Postimplantation dosimetry was performed with the GENIE treatment planning system v1.0.4 (Nucletron, Netherland), and included calculation of dose to the "classical" Point A bilaterally (a reference location $2 \mathrm{~cm}$ lateral and 2 $\mathrm{cm}$ superior to the cervical os), pelvic sidewall bilaterally (Point $\mathrm{P}$, defined as the point $2 \mathrm{~cm}$ above the top of the colpostat and $6 \mathrm{~cm}$ lateral to midline), and the rectal point and bladder point as defined by the International Commission on Radiation Units and Measurements [19]. For each implant, point doses to Points A and P, the bladder point, and the rectal point were recorded; after completion of therapy, the doses for the six implants were summed. There is no standard or universally accepted fraction size for HDR brachytherapy. At our institution we have chosen to use the fraction size of $500 \mathrm{cGy}$.

\section{Conventional treatment planning for comparison}

Conventional whole pelvic radiation therapy (WPRT) plans were generated using Pinnacle3 treatment planning system (Philips Healthcare, Madison, Wisconsin, USA). The isocenter was placed at the geometric center of the PTV. A 4-field "box" plan was designed using 6-MV photons with apertures shaped to the PTV in each beam's eyeview. The pelvic field extended from the upper margin of L5 to the midportion of the obturator foramen or the lowest level of disease, with a 2-cm margin, and laterally 1.5 $\mathrm{cm}$ beyond the lateral margins of the bony pelvic wall (at least $7 \mathrm{~cm}$ from the midline). For the lateral fields, the anterior border was the pubic symphysis and the posterior border was the space between S2 and S3. The fields could be modified to include areas of known tumor and wedges were used as needed. All plans were normalized to cover $98 \%$ of the PTV with $50.4 \mathrm{~Gy}$. The $2 \%$ underdose represents those voxels at the periphery. This normalization provided conformal coverage while minimizing dose nonuniformity within the target.

\section{Dose-volume analysis of treatment plans}

Dose-volume histograms (DVHs) of the PTVs and the critical normal structures were analyzed accordingly. For 
PTVs, we evaluated the volume, the volume covered by $95 \%$ of the prescription dose (V95), and the minimum doses delivered to $5 \%\left(D_{5}\right)$ and $95 \%\left(D_{95}\right)$ of the PTV. The critical organs with functional subunits organized in a series were examined. The conformal index (CI) and the uniformity index (UI) had been used to evaluate the conformity and uniformity of the plan. The volume received the mean dose for PTV generated from the DVH. The conformal index (CI) for PTV was calculated using the formula $\mathrm{CI}_{\mathrm{ICRU}}=V_{T V} / V_{P T V}$, where $V_{T V}$ was the ratio of the treated volume enclosed by the prescription isodose surface and $V_{P T V}$ was the planning target volume [17]. The uniformity index (UI) was defined as $\mathrm{UI}=D_{5} / D_{95}$, where $\mathrm{D}_{5}$ and $\mathrm{D}_{95}$ were the minimum doses delivered to $5 \%$ and $95 \%$ of the PTV reported previously [20].

\section{Toxicity}

Interruptions in radiotherapy might be necessitated by uncontrolled diarrhea, or other acute complications. If radiation therapy was held, then chemotherapy would also be held. Chemotherapy stopped at the completion of RT. If chemotherapy was held, radiation therapy would continue. Radiation was only stopped in cases of grade 4 hematologic or non-hematologic toxicity until toxicity resolved to at least grade 3. CDDP was withheld in any case involving grade 3 toxicity until the toxicity regressed to any grade of $<3$; in patients with grade 3 toxicity that persisted $>2$ weeks, chemotherapy was no longer administered.

\section{Follow-up}

Upon treatment completion, patients were evaluated every 3 months for the first year, every 4 months during the second year, every 6 months during the third year, and annually thereafter. At each visit, a physical and pelvic examination, blood counts, clinical chemistry, and chest x-rays were performed. Computed tomography (CT) scan, ultrasound (US), and other imaging studies were conducted when appropriate. Suspected cases of persistent or recurrent disease were confirmed by biopsy whenever possible. Acute and late toxicities (occurring $>90$ days after beginning RT) were defined and graded according to the Common Terminology Criteria for Adverse Events v3.0 (CTCAE v3.0).

\section{Statistical methods}

Descriptive statistics (mean, median, proportions) were calculated to characterize the patient, disease, and treatment features as well as toxicities after treatment. The overall survival (OS), progression-free survival (PFS), locoregional progression-free (LRPF), and distant metastases-free (DMF) rates were estimated using the KaplanMeier product-limit method. Progression was defined as a 50 percent increase in the product of the two largest diameters of the primary tumor or metastasis. Progression-free survival was calculated from the date of pathologic proof to the date of the first physical or radiographic evidence of disease progression, death, or the last follow-up visit. Survival was calculated from the date of pathologic proof to the date of death or the last follow-up visit. All analyses were performed using the Statistical Package for the Social Sciences, version 12.0 (SPSS, Chicago, IL, USA).

\section{Results \\ Patient characteristics}

Ten women were included. They had a median age of 58 years (range, 33-72 years). All belong to FIGO Stage IIB and IIIB. The medium tumor volume was $45.9 \mathrm{~cm}^{3}$. The medium weekly cycles of chemotherapy were 5 weeks. Seventy percent of patients could complete 4 weekly cycles of chemotherapy. All of the patients were treated with definitively concurrent chemotherapy with WPHT followed by brachytherapy. (Table 1 )

\section{Treatment outcome}

The mean survival was 25 months (range, 3 to 27 months). The actuarial 2-year overall survival, progressfree survival, locoregional control and distant metastasisfree rates were $67 \%, 77 \%, 90 \%$ and $88 \%$, respectively. The 2-year survival, progression-free, locoregional-progression-free and distant metastasis-free patient number over all patients are $9 / 10,8 / 10,9 / 10$ and 9/10, respectively. Ninety percent of patients were surviving at the time of this report.

\section{Dose-volume analysis and comparison for WPHT and WPRT}

The WPHT for UI and CI was $1.07 \pm 0.05$ and $1.01 \pm 0.05$, respectively. The UI and $\mathrm{CI}$ for individual patient are plotted in Figures 1A and 1B, respectively. Dose-volume histograms statistics for the organs at risk are described in table 2. WPHT provided better critical organs sparing than WPRT in the mean dose and the other parameters for rectum, bladder and intestine with a statistically significant level ( $p$ value $<0.01$ ), respectively. WPHT provided impressive ability of high dose declining for OARs than WPRT. However, WPHT had poorer results for right and left side pelvic bone sparing than WPRT due to lacking of V10 and V20 constraint for planning initially.

\section{Acute and subacute toxicity}

Acute toxicity of radiation therapy within chemotherapy and late toxicity is detailed in Additional file 1. One grade 3 of acute toxicity for diarrhea, thrombocytopenia and three grade 3 of leucopenia were noted during CCRT. Only one grade 3 of subacute toxicity for thrombocytopenia was noted. There was no grade 3 or 4 subacute toxicities for anemia, leucopenia, genitourinary or gastrointestinal. 
Table I: Patient characteristics

\begin{tabular}{|c|c|}
\hline Variable & No. of patient (\%) \\
\hline \multicolumn{2}{|l|}{ Age (years) } \\
\hline Median (range) & $58(33-72)$ \\
\hline \multicolumn{2}{|l|}{ Gender } \\
\hline Female & $\begin{array}{c}10 \\
(100 \%)\end{array}$ \\
\hline \multicolumn{2}{|l|}{ Karnofsky performance status } \\
\hline$<70$ & 0 \\
\hline$\geq 70$ & $\begin{array}{c}10 \\
(100 \%)\end{array}$ \\
\hline \multicolumn{2}{|l|}{ Pathology } \\
\hline Squamous cell carcinoma & $\begin{array}{c}7 \\
(70 \%)\end{array}$ \\
\hline Adenocarcinoma & $\begin{array}{c}3 \\
(30 \%)\end{array}$ \\
\hline \multicolumn{2}{|c|}{ International Federation of Gynecology and Obstetrics (FIGO) stage } \\
\hline Stage IIB & $\begin{array}{c}9 \\
(90 \%)\end{array}$ \\
\hline Stage IIIB & $\begin{array}{c}1 \\
(10 \%)\end{array}$ \\
\hline \multicolumn{2}{|l|}{ Tumor size } \\
\hline Medium length (range) & $\begin{array}{c}5.5 \mathrm{~cm} \\
(4.3-8.4 \mathrm{~cm})\end{array}$ \\
\hline Medium depth (range) & $\begin{array}{c}3.7 \mathrm{~cm} \\
(2.4-4.6 \mathrm{~cm})\end{array}$ \\
\hline Medium width (range) & $\begin{array}{c}4.4 \mathrm{~cm} \\
(3.5-6.0 \mathrm{~cm})\end{array}$ \\
\hline \multicolumn{2}{|l|}{ Weekly cycles of chemotherapy } \\
\hline 5 weeks & $\begin{array}{c}5 \\
(50 \%)\end{array}$ \\
\hline 4 weeks & $\begin{array}{c}2 \\
(20 \%)\end{array}$ \\
\hline 3 weeks & $\begin{array}{c}1 \\
(10 \%)\end{array}$ \\
\hline 2 weeks & $\begin{array}{c}2 \\
(20 \%)\end{array}$ \\
\hline
\end{tabular}

\section{Discussion}

In our preliminary results of locally advanced cervical cancer receiving WPHT concurrent with chemotherapy followed by brachytherapy, HT provides feasible outcomes and acceptable toxicity during and after CCRT.

The 2-year estimate of OS, PFS, locoregional failure only and distant metastasis only rate in the RT plus weekly CDDP reported by randomized trials was $67-71 \%, 64$ $84 \%, 10-25 \%$ and $6-11 \%$, respectively $[2,3,21]$. The overall survival, disease-free survival, locoregional failure and distant metastasis rate at 2 years in our institute are $67 \%, 77 \%, 10 \%$ and $12 \%$, respectively. The clinical results of WPHT concurrent with weekly CDDP following by HDR brachytherapy at our institute suggest WPHT is feasible for locally advanced cervical carcinoma patients.

Adding more beams would lead to improved conformality without affecting the value of the objective function [20]. The CI is usually larger than 1 , indicating that a por- tion of the prescription dose was delivered outside the PTV. The greater the CI, the less is the dose conformity to the PTV [20]. The greater UI indicates higher heterogeneity in the PTV [22]. In the current study, the UI and CI for WPHT was $1.07 \pm 0.05$ and $1.01 \pm 0.05$, respectively. WPHT provides the impressed conformality and uniformity for locally advanced cervical carcinoma patients. The $\mathrm{UI}$ and $\mathrm{CI}$ for individual patient are described in Fig. 1A and $1 \mathrm{~B}$, respectively.

Despite the clear efficacy of a combined modality approach in locally advanced cervical cancers $[2,3,21]$, toxicity can be considerable. For locally advanced cervical cancer treated with CCRT, the rates of grade 3 acute toxicities for GI effects were 7 - 9\% [2,3,23]. For moderate acute hematologic effects, the happening rate during CCRT was reported from $23 \%$ to $37 \%[2,3,23]$. In the current study, the moderate acute toxicities during CCRT are listed as follow: one $(1 / 10)$ for diarrhea, three $(3 / 10)$ for leukopenia and one $(1 / 10)$ for thrombocytopenia. (Additional file 1 ) 

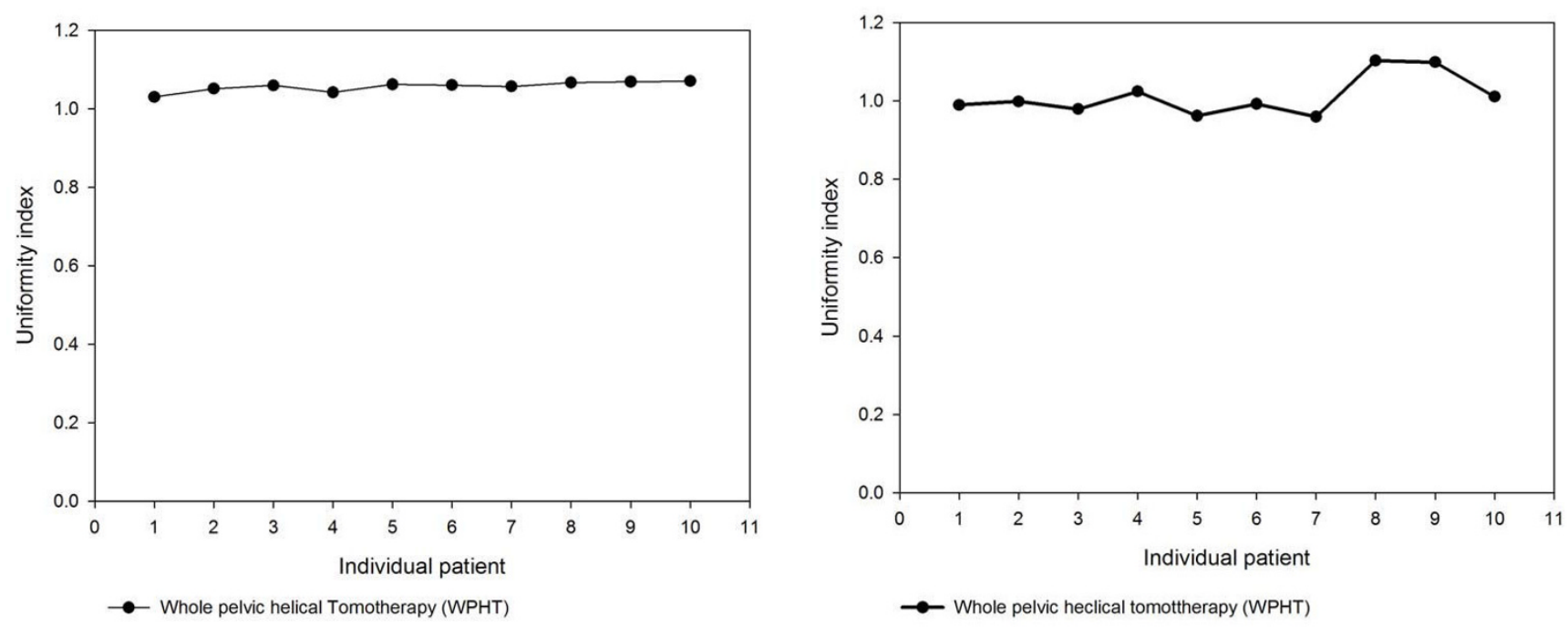

Figure I

(A) The uniformity index of helical tomotherapy for 10 patients with locally advanced cervical cancer. (B) The conformal index of helical tomotherapy for with locally advanced cervical cancer.

Table 2: Dose-volume histograms statistics for the organs at risk

Average $\pm *$ S.D.

\begin{tabular}{|c|c|c|c|c|c|}
\hline Organ & Volume $(\mathrm{ml}) \pm * S . D$. & Helical tomotherapy & Conventional radiotherapy & †Decreasing percentage & $p$ value \\
\hline Rectum & $43.5 \pm 18.2$ & & & & \\
\hline Mean dose & & $41.3 \pm 5.1 \mathrm{~Gy}$ & $50.9 \pm 1.9 \mathrm{~Gy}$ & $18.9 \%$ & $<0.01$ \\
\hline V50.4 & & $37.2 \pm 30.1 \%$ & $80.8 \pm 12.4 \%$ & $55.6 \%$ & $<0.01$ \\
\hline V40 & & $68.3 \pm 20.9 \%$ & $95.2 \pm 4.2 \%$ & $35.0 \%$ & $<0.01$ \\
\hline V30 & & $82.2 \pm 15.3 \%$ & $98.4 \pm 2.6 \%$ & $16.6 \%$ & $<0.01$ \\
\hline Bladder & $59.8 \pm 24.2$ & & & & \\
\hline Mean dose & & $40.5 \pm 3.5 \mathrm{~Gy}$ & $50.2 \pm 2.5 \mathrm{~Gy}$ & $19.3 \%$ & $<0.01$ \\
\hline V50.4 & & $29.5 \pm 14.7 \%$ & $74.4 \pm 17.6 \%$ & $61.3 \%$ & $<0.01$ \\
\hline V45 & & $49.1 \pm 13.7 \%$ & $86.0 \pm 11.5 \%$ & $43.2 \%$ & $<0.01$ \\
\hline V40 & & $57.9 \pm 12.6 \%$ & $91.3 \pm 8.5 \%$ & $36.8 \%$ & $<0.01$ \\
\hline V30 & & $75.7 \pm 12.3 \%$ & $100.0 \pm 0 \%$ & $24.3 \%$ & $<0.01$ \\
\hline Intestine & $1523.3 \pm 1389.4$ & & & & \\
\hline Mean dose & & $25.1 \pm 2.4 \mathrm{~Gy}$ & $34.2 \pm 4.2 \mathrm{~Gy}$ & $26.3 \%$ & $<0.01$ \\
\hline V50.4 & & $0.4 \pm 0.4 \%$ & $20.0 \pm 10.7 \%$ & $98.2 \%$ & $<0.01$ \\
\hline V40 & & $4.9 \pm 3.2 \%$ & $33.3 \pm 13.1 \%$ & $84.1 \%$ & $<0.01$ \\
\hline V30 & & $23.5 \pm 11.9 \%$ & $59.5 \pm 10.4 \%$ & $61.1 \%$ & $<0.01$ \\
\hline $\mathrm{V} 20$ & & $69.2 \pm 10.9 \%$ & $86.6 \pm 8.0 \%$ & $20.1 \%$ & $<0.01$ \\
\hline Right femur & $114.4 \pm 16.2$ & & & & \\
\hline V30 & & $15.5 \pm 14.2 \%$ & $23.2 \pm 29.1 \%$ & $19.0 \%$ & 0.47 \\
\hline Left femur & $1 \mid 4.3 \pm 14.0$ & & & & \\
\hline V30 & & $16.1 \pm 13.9 \%$ & $22.3 \pm 28.5 \%$ & $12.9 \%$ & 0.54 \\
\hline Left pelvic bone & $187.3 \pm 19.4$ & & & & \\
\hline VIO & & $99.9 \pm 0.1 \%$ & $93.1 \pm 4.8 \%$ & $-6.8 \%$ & $<0.01$ \\
\hline $\mathrm{V} 20$ & & $79.1 \pm 4.6 \%$ & $86.2 \pm 5.6 \%$ & $8.2 \%$ & $<0.01$ \\
\hline Right pelvic bone & $189.4 \pm 20.1$ & & & & \\
\hline VI0 & & $99.9 \pm 0.1 \%$ & $95.5 \pm 2.1 \%$ & $-4.4 \%$ & $<0.01$ \\
\hline $\mathrm{V} 20$ & & $78.3 \pm 4.8 \%$ & $89.2 \pm 3.1 \%$ & $12.2 \%$ & $<0.01$ \\
\hline
\end{tabular}

*S.D.: standard deviation.

† Decreasing percentage: (conventional radiotherapy - helical tomotherapy)/conventional radiotherapy 


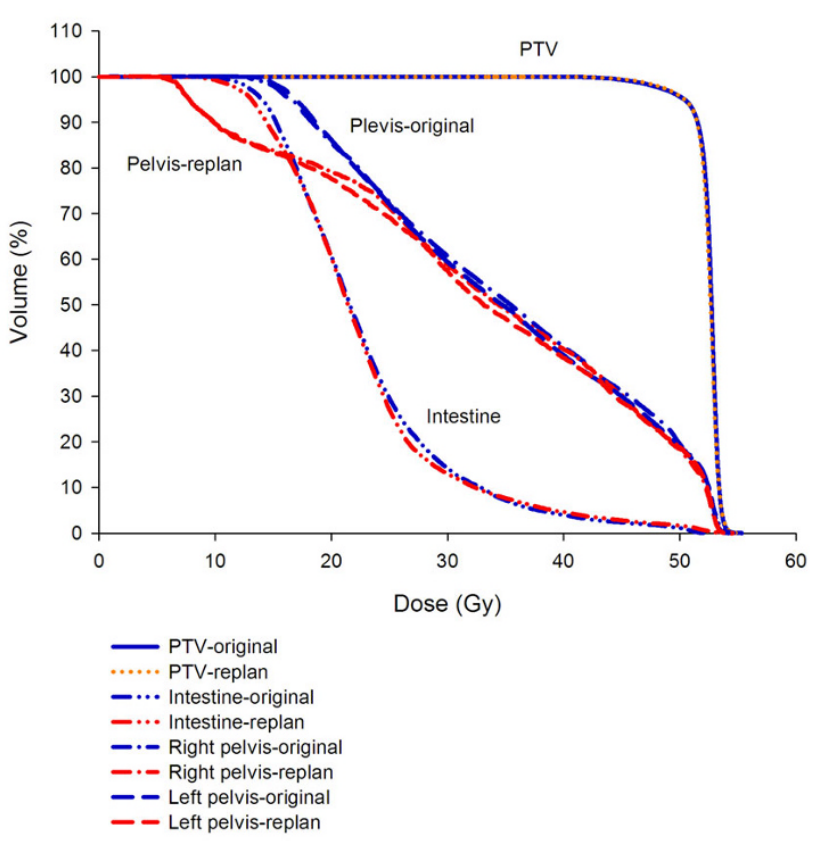

Figure 2

Dose-volume histogram of pelvic bone marrow under the similar PTV and intestine dose for one patient with original whole pelvic helical tomotherapy and giving VI $0<90 \%$, V20 $<80 \%$ replanning whole pelvic helical tomotherapy for comparisons.

The acute toxicities of GI and GU for locally advanced cervical cancer treated by WPHT are feasible however the dominant hematologic toxicities are noted in the current study. The late moderate toxicities for locally advanced cervical cancer patients treated with CCRT that reported by previous series are 9.4 - 13\% for GI effects and 3 $14.5 \%$ for genitourinary effects $[5,21,23]$. In the current study, the subacute grade 3 toxicity is only $1(10 \%)$ for thrombocytopenia and there are none with GI and GU effects. (Additional file 1) Compared with WPRT, WPHT decreases the mean dose to rectum, bladder and intestines successfully. In addition, the V50 decreasing percentage for WPHT in rectum, bladder and intestine is 56\%, 61\% and $98 \%$, respectively. (Table 2 ) From the view of physics, WPHT decreases the mean and high doses to the OARs entirely when compared with conventional technique and these physic properties of WPHT reflect the declining rate of acute and subacute toxicities for gastrointestinal and genitourinary events successfully. (Additional file 1)

There are numbers of groups that explored how IMRT can minimize the gastrointestinal, genitourinary and bone marrow toxicity than conventional RT for gynecologic cancer patients. When using IMRT techniques for gynecologic treatment, V40 and V30 for the intestine, bladder and rectum is $25-40 \%$ and $40-57 \%, 65-86 \%$ and 88 97\%, $74-84 \%$ and 87 - 95\%, respectively [24-27]. (Additional file 2) Compared with previous reports, HT decreases $80-88 \%$ of V40 and $40-60 \%$ of V30 for the intestine, $11-33 \%$ of V40 and $14-22 \%$ of V30 for the bladder and 8 - 19\% of V40 and 6 - 14\% of V30 for the rectum than previous IMRT reports, respectively. It also notes that HT decreases 35\% of V45 for the intestine than previous IMRT reports simultaneously. In other words, HT provides significantly superiority for decreasing high dose to these OARs than IMRT does. Therefore, we suggest when treating the locally advanced cervical cancer patients with HT, the optimization constraints of V40 and V30 for the intestine, bladder and rectum could be reconsidered as $5 \%$ and $24 \%, 58 \%$ and $76 \%, 68 \%$ and $82 \%$, respectively.

HT can deliver dose to bone marrow exactly in total marrow irradiation and reduce the dose to OARs around 51\%$74 \%$ when compared with total body irradiation [13]. It implies that HT can manage bone marrow precisely, either targeting or sparing. Brixey et al. [8] reported that acute hematological toxicity was reduced with pelvic IMRT compared with four-field box techniques in gynecologic cancer patients undergoing chemotherapy. Mell et al. [28] also provided evidence of an association between the volume of pelvic BM receiving low-dose radiation (V10, V20) and pointed out the potential of bone marrow sparing-IMRT could diminish the chronic effects of RT on BM suppression, improving chemotherapy tolerance. In our study, the pelvic bones sparing technique did not perform in the original WPHT plan and the value of V10 for pelvic bones almost achieving $100 \%$ was noted. In our retrospective data, $40 \%$ of acute moderate hematological toxicities happened in the CCRT and 10\% of subacute thrombocytopenia was noted in the following days. It is noted that the highly conformal doses distribute to target and large off-target low dose existing simultaneously in the HT plan. If we target pelvic bone marrow according to Brixey et al. [8] and set pelvic bone marrow optimal constraints directly, HT can provide as much bone marrow sparing in the low dose as we desired. (Fig. 2) Since June first, the following cervical cancer patients in our center were performed pelvic bone sparing technique with WPHT. Up to day, three locally advanced cervical cancer patients completed the treatment by WPHT concurrent with chemotherapy and only grade 1 or 2 acute hematologic toxicities during CCRT are noted. The encouraging results hints that targeting pelvic bones and setting optimal constraint for pelvic bones can potentially decrease the acute and subacute clinical toxicities when use WPHT.

There are some limitations in our current study. First, the small case number and the retrospective study design make any statistical conclusions very tentative. Second, the follow-up time is short so the long-term results need 
to keep closely follow-up. Third, we do not perform pelvic bones sparing within this study perhaps this is the reason for acute hematologic toxicities dominant therefore enroll more patients by limiting bone marrow radiation dose with WPHT technique in the future to confirm our observation is warranted.

\section{Conclusions}

To sum up, whole pelvic helical tomotherapy provides feasible clinical results in patients with locally advanced cervical carcinoma. Long-term follow-up and to enroll more locally advanced cervical carcinoma patients by limiting bone marrow radiation dose with WPHT technique is warranted.

\section{Competing interests}

We have no personal or financial conflict of interest and have not entered into any agreement that could interfere with our access to the data on the research, or upon our ability to analyze the data independently, to prepare manuscripts, and to publish them.

\section{Authors' contributions}

All authors read and approved the final manuscript. $\mathrm{CHH}$ and PWS carried out all CT evaluations, study design, target delineations and interpretation of the study. $\mathrm{CHH}$ drafted the manuscript. MCW, SMH and CAC took care of cervical cancer patients. HYL made the treatment planning and carried out all WPHT and WPRT comparisons and evaluations. THT and YJC participated in manuscript preparation and study design. LYW and YPH gave advice on the work and carried out statistical analysis.

\section{Additional material}

\section{Additional file 1}

Acute and subacute toxicity for locally advanced cervical cancer patients received chemotherapy concurrent with whole pelvic helical tomotherapy followed by brachytherapy.

Click here for file

[http://www.biomedcentral.com/content/supplementary/1748717X-4-62-S1.DOC]

\section{Additional file 2}

The rate of cervical carcinoma treated with concurrent chemoradiation using helical tomotherapy at the Far Eastern Memorial Hospital (FEMH) compared with selected published series.

Click here for file

[http://www.biomedcentral.com/content/supplementary/1748717X-4-62-S2.DOC]

\section{Acknowledgements}

We are indebted to Wei-Hsiang Kung, M.S. for the data collection.

\section{References}

I. Sundar SS, Horne A, Kehoe S: Cervical cancer. Clin Evid (Online) 2008, 2008:08I8.

2. Rose PG, Bundy BN, Watkins EB, Thigpen JT, Deppe G, Maiman MA, Clarke-Pearson DL, Insalaco S: Concurrent cisplatin-based radiotherapy and chemotherapy for locally advanced cervical cancer. N Engl J Med 1999, 340: I I 44-53.

3. Whitney CW, Sause W, Bundy BN, Malfetano JH, Hannigan EV, Fowler WC Jr, Clarke-Pearson DL, Liao SY: Randomized comparison of fluorouracil plus cisplatin versus hydroxyurea as an adjunct to radiation therapy in stage IIB-IVA carcinoma of the cervix with negative para-aortic lymph nodes: a Gynecologic Oncology Group and Southwest Oncology Group study. J Clin Oncol 1999, 17:1339-48.

4. John M, Flam M, Caplan R, Rotman M, Quivey J, Steinfeld A, Russell $A$ : Final results of a phase II chemoradiation protocol for locally advanced cervical cancer: RTOG 85-15. Gynecol Oncol 1996, 61:221-26.

5. Tan LT, Zahra M: Long-term survival and late toxicity after chemoradiotherapy for cervical cancer--the Addenbrooke's experience. Clin Oncol (R Coll Radiol) 2008, 20:358-64.

6. Mundt AJ, Lujan AE, Rotmensch J, Waggoner SE, Yamada SD, Fleming G, Roeske JC: Intensity-modulated whole pelvic radiotherapy in women with gynecologic malignancies. Int J Radiat Oncol Biol Phys 2002, 52: 1330-37.

7. Portelance L, Chao KS, Grigsby PW, Bennet H, Low D: Intensitymodulated radiation therapy (IMRT) reduces small bowel, rectum, and bladder doses in patients with cervical cancer receiving pelvic and para-aortic irradiation. Int J Radiat Oncol Biol Phys 200I, 5 I:26I-66.

8. Brixey CJ, Roeske JC, Lujan AE, Yamada SD, Rotmensch J, Mundt AJ: Impact of intensity-modulated radiotherapy on acute hematologic toxicity in women with gynecologic malignancies. Int J Radiat Oncol Biol Phys 2002, 54: I388-96.

9. Lujan AE, Mundt AJ, Yamada SD, Rotmensch J, Roeske JC: Intensitymodulated radiotherapy as a means of reducing dose to bone marrow in gynecologic patients receiving whole pelvic radiotherapy. Int J Radiat Oncol Biol Phys 2003, 57:516-2I.

10. Ahmed RS, Kim RY, Duan J, Meleth S, De Los Santos JF, Fiveash JB: IMRT dose escalation for positive para-aortic lymph nodes in patients with locally advanced cervical cancer while reducing dose to bone marrow and other organs at risk. Int J Radiat Oncol Biol Phys 2004, 60:505-12.

II. Bunt $L$ van de, Heide UA van der, Ketelaars M, de Kort GA, Jurgenliemk-Schulz IM: Conventional, conformal, and intensity-modulated radiation therapy treatment planning of external beam radiotherapy for cervical cancer: The impact of tumor regression. Int J Radiat Oncol Biol Phys 2006, 64:189-96.

12. Shueng PW, Wu LJ, Chen SY, Hsiao CH, Tien HJ, Cheng PW, Kuo YS Chen YJ, Chen CA, Hsieh PY, Hsieh CH: Concurrent Chemoradiation Therapy with Helical Tomotherapy for Oropharyngeal Cancer - A Preliminary Result. Int J Radiat Oncol Biol Phys 2009 in press.

13. Shueng PW, Lin SC, Chong NS, Lee HY, Tien HJ, Wu LJ, Chen CA, Lee J, Hsieh CH: Total marrow irradiation with helical tomotherapy for bone marrow transplantation of multiple myeloma: first experience in Asia. Technol Cancer Res Treat 2009, 8:29-38.

14. Esthappan J, Chaudhari S, Santanam L, Mutic S, Olsen J, Macdonald DM, Low DA, Singh AK, Grigsby PW: Prospective clinical trial of positron emission tomography/computed tomography image-guided intensity-modulated radiation therapy for cervical carcinoma with positive para-aortic lymph nodes. Int J Radiat Oncol Biol Phys 2008, 72: I I34-39.

15. Benedet JL: Editorial. Int J Gynaecol Obstet 2000, 70:207-08.

16. ICRU: International Commission on Radiation Units and Measurements (ICRU). Prescribing, recording, and reporting photon beam therapy, ICRU report 50: Bethesda, MD: ICRU I 993 [http:// www.oxfordjournals.org/jicru/backissues/reports.html].

17. ICRU: International Commission on Radiation Units and Measurements (ICRU). Prescribing, Recording and Reporting Photon Beam Therapy (Supplement to ICRU Report 50), ICRU Report, 62. Bethesda, MD: ICRU 1999 [http://www.oxfordjournals.org/jicru/back issues/reports.html].

18. Forrest LJ, Mackie TR, Ruchala K, Turek M, Kapatoes J, Jaradat H, Hui S, Balog J, Vail DM, Mehta MP: The utility of megavoltage com- 
puted tomography images from a helical tomotherapy system for setup verification purposes. Int J Radiat Oncol Biol Phys 2004, 60:1639-44.

19. ICRU: International Commission on Radiation Units and Measurements (ICRU). Dose and volume specification for reporting intracavitary therapy in gynecology, ICRU Report, 38. Bethesda, MD: ICRU 1985 [http://www.oxfordjournals.org/jicru/backissues/reports.html].

20. Wang X, Zhang X, Dong L, Liu H, Gillin M, Ahamad A, Ang K, Mohan R: Effectiveness of noncoplanar IMRT planning using a parallelized multiresolution beam angle optimization method for paranasal sinus carcinoma. Int J Radiat Oncol Biol Phys 2005, 63:594-601.

21. Eifel PJ, Winter K, Morris M, Levenback C, Grigsby PW, Cooper J, Rotman M, Gershenson D, Mutch DG: Pelvic irradiation with concurrent chemotherapy versus pelvic and para-aortic irradiation for high-risk cervical cancer: an update of radiation therapy oncology group trial (RTOG) 90-0 I. J Clin Oncol 2004, 22:872-80.

22. Chen ZY, Ma YB, Sheng XG, Zhang XL, Xue L, Song QQ, Liu NF, Miao $\mathrm{HQ}$ : [Intensity modulated radiation therapy for patients with gynecological malignancies after hysterectomy and chemotherapy/radiotherapy]. Zhonghua Zhong Liu Za Zhi 2007, 29:305-08.

23. Morris M, Eifel PJ, Lu J, Grigsby PW, Levenback C, Stevens RE, Rotman $M$, Gershenson DM, Mutch DG: Pelvic radiation with concurrent chemotherapy compared with pelvic and paraaortic radiation for high-risk cervical cancer. $N$ Engl J Med 1999, 340: I I37-43.

24. Georg P, Georg D, Hillbrand M, Kirisits C, Potter R: Factors influencing bowel sparing in intensity modulated whole pelvic radiotherapy for gynaecological malignancies. Radiother Oncol 2006, 80:19-26.

25. Menkarios C, Azria D, Laliberte B, Moscardo CL, Gourgou S, Lemanski C, Dubois JB, Ailleres N, Fenoglietto P: Optimal organ-sparing intensity-modulated radiation therapy (IMRT) regimen for the treatment of locally advanced anal canal carcinoma: a comparison of conventional and IMRT plans. Radiat Oncol 2007, 2:4I.

26. Mell LK, Tiryaki H, Ahn KH, Mundt AJ, Roeske JC, Aydogan B: Dosimetric comparison of bone marrow-sparing intensity-modulated radiotherapy versus conventional techniques for treatment of cervical cancer. Int J Radiat Oncol Biol Phys 2008, 7I:I504-I0.

27. Roeske JC, Lujan A, Rotmensch J, Waggoner SE, Yamada D, Mundt AJ: Intensity-modulated whole pelvic radiation therapy in patients with gynecologic malignancies. Int J Radiat Oncol Biol Phys 2000, 48:1613-21.

28. Mell LK, Kochanski JD, Roeske JC, Haslam JJ, Mehta N, Yamada SD, Hurteau JA, Collins YC, Lengyel E, Mundt AJ: Dosimetric predictors of acute hematologic toxicity in cervical cancer patients treated with concurrent cisplatin and intensity-modulated pelvic radiotherapy. Int J Radiat Oncol Biol Phys 2006, 66: I356-65.
Publish with Biomed Central and every scientist can read your work free of charge

"BioMed Central will be the most significant development for disseminating the results of biomedical research in our lifetime. "

Sir Paul Nurse, Cancer Research UK

Your research papers will be:

- available free of charge to the entire biomedical community

- peer reviewed and published immediately upon acceptance

- cited in PubMed and archived on PubMed Central

- yours - you keep the copyright

Submit your manuscript here:

http://www.biomedcentral.com/info/publishing_adv.asp
BioMedcentral 